\title{
Gestão de custos no setor público: um panorama de experiências internacionais
}

\author{
Diego Messias \\ Universidade Estadual do Oeste do Paraná (Unioeste) \\ Júlio César Ferreira \\ Universidade Estadual do Oeste do Paraná (Unioeste)
}

Dione Olesczuk Soutes

Universidade Estadual do Oeste do Paraná (Unioeste)

No contexto de reformas gerenciais ocorridas na administração pública nos últimos anos, este artigo objetivou investigar experiências internacionais relativas à implementação de sistemas e ferramentas de gestão de custos, de forma a identificar as principais dificuldades enfrentadas pelos entes governamentais no processo, além dos benefícios proporcionados à gestão. Por meio de bibliometria e análise de conteúdo, foram visitados 17 artigos internacionais que apresentavam cases de implantação ou discutiam a temática sob outra ótica e metodologia. Os resultados indicam que os projetos de adoção de técnicas de gerenciamento de custos são dificultados pela não integração com os demais sistemas estruturantes organizacionais e com o planejamento estratégico; escassez de recursos para viabilização e manutenção dos projetos; alta burocratização e normatização características do setor público. Também se constatou que a busca por legitimação social por parte dos gestores também causa prejuízos à gestão pública.

Palavras-chave: contabilidade de custos, implementação, administração pública

[Artigo recebido em 26 de outubro de 2017. Aprovado em 18 de maio de 2018.] 


\section{Gestión de costos en el sector público: un panorama de experiencias internacionales}

En el contexto de reformas gerenciales ocurridas en la administración pública en los últimos años, este artículo objetivó investigar experiencias internacionales relativas a la implementación de sistemas y herramientas de gestión de costos, para identificar las principales dificultades enfrentadas por los entes gubernamentales en el proceso, además de los beneficios proporcionados a la gestión. Por medio de bibliometría y análisis de contenido, fueron visitados 17 artículos internacionales que presentaron casos de implantación o discutieron la temática bajo otra óptica y metodología. Los resultados indican que los proyectos de adopción de técnicas de gestión de costos se ven dificultados por la no integración con los demás sistemas estructurantes organizativos y con la planificación estratégica, escasez de recursos para viabilización y mantenimiento de los proyectos, alta burocratización y normatización características del sector público. También se constató que la búsqueda por legitimación social por parte de los gestores también causa perjuicios a la gestión pública.

Palabras clave: contabilidad de costes, implementación, administración pública

\section{Cost management in the state sector: international experiences in outlook}

In the context of managerial reforms in public administration in recent years, this article aimed to investigate international experiences regarding the implementation of cost management systems and tools, in order to identify the main difficulties faced by government entities in the process, in addition to the benefits provided to management. Through bibliometrics and content analysis, 17 international papers were visited that presented cases of implantation or discussed the theme from another point of view and methodology. The results indicate that projects for the adoption of cost management techniques are hampered by non integration with other organizational structuring systems and strategic planning, scarce resources for project feasibility and maintenance, high bureaucratization and standardization of the public sector. It was also found that the search for social legitimacy on the part of the managers also causes damages to the public management.

Keywords: cost accounting, implementation, public administration 


\section{Introdução}

Para atingimento de sua função, o Estado, ao planejar suas ações, deve fixar critérios para o desenvolvimento de soluções às diversas demandas sociais. Para o alcance da eficiência, eficácia e efetividade dos serviços prestados, se faz necessária a adoção de ferramentas de gestão e controle organizacional que otimizem a destinação dos recursos públicos despendidos pela sociedade (TER BOGT, 2008). Uma das principais funções da administração pública é justamente o emprego adequado dos recursos arrecadados em favor do bem-estar social (LAPSLEY; WRIGHT, 2004; LOVATO et al., 2016), e os cidadãos esperam da gestão o melhor atendimento possível de suas demandas sociais, pelo uso eficiente desses recursos, além da transparência dos atos praticados (MOTTA, 2013).

O orçamento público, como principal ferramenta gerencial adotada pelo governo brasileiro, atua predominantemente em ações de controle, não medindo, e tampouco garantindo, o nível de eficiência esperado dos gestores. Buscando sanar essa lacuna, por meio do monitoramento e da responsabilização dos gestores pelos atos por eles praticados, ao menos em parte, foi editada Lei de Responsabilidade Fiscal (LRF) - Lei Complementar no 101/2000.

Além da responsabilização, a LRF instituiu uma série de instrumentos importantes para o avanço econômico e social e necessários para aprimorar ferramentas que permitam à sociedade captar dados quantitativos sobre a execução dos programas de governo. Esses dados dão condições, aos cidadãos, para avaliar a eficiência e a eficácia da gestão da administração pública e, assim, ter subsídios consistentes para cobrar as melhorias necessárias (LOVATO et al., 2016; ModELL, 2009). Uma das ferramentas estabelecidas pela LRF é a obrigatoriedade de implementação de sistemas de gerenciamento de custos (BRASIL, 2000; FrAnco et al., 2013), até então inexistente na administração pública brasileira.

Outro fator que colaborou para a implementação do sistema de custos no setor público brasileiro foi a Portaria no 184/2008 do Ministério da Fazenda, que atribuiu ao Conselho Federal de Contabilidade a tarefa de emitir as Normas Brasileiras de Contabilidade Aplicadas ao Setor Público (NBCASP); e à Secretaria do Tesouro Nacional, a missão de desenvolver ações no sentido de promover a convergência para os padrões internacionais. Nesse cenário nasceu a NBCASP 16.11, que estabelece as regras básicas para mensuração e evidenciação dos custos no setor público.

A utilização da informação de custos, como ferramenta de controle e como instrumento estratégico, ganha relevância no contexto social atual, em que os governantes são cada vez mais exigidos por uma administração mais eficiente dos 
recursos públicos (OSBORNE; GAEBLER, 1992). Essas informações podem apresentar, como destacam Lovato et al. (2016), por exemplo, custo por aluno (em escolas ou universidades) e custo por paciente (em hospitais). Ademais, os autores ressaltam que esses dados permitem a medição da eficiência do gasto, ao possibilitar sua comparação com custos de outros entes governamentais e também com os valores praticados pela iniciativa privada.

Todavia, para que se chegue ao cenário de usabilidade da informação de custos, há um árduo e sinuoso caminho a ser percorrido: a implementação do sistema e todo seu aparato na máquina pública. Cardoso, Aquino e Bitti (2011) alertam que a implantação em massa (na administração pública) do sistema de informações de custos somente se tornará realidade a partir do momento em que os potenciais usuários da informação produzida pelo sistema percebam sua relevância e tomem as medidas necessárias para a concretização do processo. Não basta a exigência legal e normativa, pois a efetiva adoção será promovida pela utilidade percebida pelos gestores (AgUILÀ, 2016; CARDoso; AQUINO; BITTI, 2011; MäTtÖ; SIPPOLA, 2016; Verbeeten, 2011).

Pesquisas científicas tendem a contribuir com processos inovadores como esse, ao destacar casos de sucesso e percepções sobre perspectivas diferenciadas. Todavia, no caso da gestão de custos no setor público, os estudos nacionais não trouxeram contribuições significativas acerca de experiências (BITTI; AQUINO; CARDOSO, 2011; FRANCO et al., 2013; SANTOS, 2015), sobretudo quanto ao processo de implantação, que é o ponto crucial e que enfrenta uma série de barreiras e dificuldades. O estudo bibliométrico de Bitti, Aquino e Cardoso (2011) confirma a falta de contribuições significativas nas pesquisas brasileiras.

Diante desse cenário, e considerando o fato de que a pesquisa nos Estados Unidos e Europa se encontra em estágio mais avançado que a nacional, conforme destacado por Bitti, Aquino e Cardoso (2011), emerge a seguinte inquietação: como se deu o processo de implementação de sistemas de gerenciamento de custos na administração pública em âmbito internacional?

Nesse contexto, tem-se como objetivo do presente trabalho investigar experiências de implementação de sistemas de gestão de custos no setor público em âmbito internacional, de forma a identificar as principais dificuldades enfrentadas pelos entes governamentais no processo, bem como os benefícios proporcionados à gestão.

A pesquisa se justifica pelo fato de as atividades de controle e gestão de custos se encontrarem em fase de implementação na administração pública brasileira, e porque a temática tem sido constante em pesquisas nacionais, sendo que a percepção do discutido em âmbito global tende a propiciar avanços à ciência e à gestão pública brasileira. 
Além desta introdução, o trabalho está estruturado por uma revisão da literatura, a descrição dos procedimentos metodológicos, análise e discussão dos trabalhos encontrados e considerações finais.

\section{Revisão de literatura}

\section{Nova Gestão Pública}

Nas últimas décadas, o setor público tem se caracterizado por profunda reestruturação em seus serviços e, principalmente, em sua gestão. O Estado passa a ter um perfil mais próximo ao encontrado no setor privado (quasimarket), passando a adotar lógicas de mercado e modelos de gestão nos quais prevalece a ênfase em resultados, que, no caso da administração pública, se dá por meio de produtos e serviços voltados para os interesses da sociedade (LAPSLEY; WRIGHT, 2004; MAGRO; PINTO, 2012; MÄTTÖ; SIPPOLA, 2016).

A reestruturação, oriunda da denominada Nova Gestão Pública (NGP) - conjunto de reformas paradigmáticas governamentais introduzido por países anglo-saxões na década de 1970 -, trouxe para a gestão pública características e práticas gerenciais empresariais como a descentralização administrativa, artefatos de planejamento estratégico, gestão de custos, controladoria, gestão de ativos intangíveis, entre outros. Modell (2009) e Ikeanyibe (2016) observaram que a NGP tem sido mais adotada nos últimos anos por países em desenvolvimento, imitando as políticas das nações desenvolvidas. Entre os fatores motivadores para a implementação das novas práticas estão a busca por relações comerciais com as grandes economias, a privatização de parte dos serviços públicos e as pressões sociais para otimização da gestão e da destinação dos recursos (IKEANYIBE, 2016; LAPSLEY; WRIGHT, 2004; LUTILSKY; VAŠIČEK; VAŠIČEK, 2012; OSBORNE; GAEBLER, 1992; MÄTTÖ; SIPPOLA, 2016; TER BOGT, 2008; VERBEETEN, 2011).

Em estudo realizado no setor público alemão, Ter Bogt (2008) identificou, todavia, vestígios de descontentamento quanto às reformas na gestão governamental por parte de colaboradores públicos. O descontentamento se originou, segundo o autor, em virtude de algumas propostas de mudanças não serem tão práticas e aplicáveis quanto esperado, e, por esse motivo, não atingirem a eficiência esperada e objetivada.

São diversas as explicações para adoção de uma ferramenta gerencial que não seja aplicada de forma integral posteriormente. Johansson e Siverbo (2009) detectaram três possíveis explicações em pesquisa acerca do governo sueco. Em primeiro lugar, citam a divergência entre os objetivos dos vários stakeholders (população em geral, 
bancadas, órgãos de classe, órgãos regulamentadores). Em segundo lugar, o fato de a adoção da ferramenta ter como objetivo apenas a legitimação política ou a imitação de outras entidades. Por último, a insuficiência de recursos financeiros, humanos e de infraestrutura necessários para implementação completa e manutenção da metodologia de gestão. Ter Bogt (2008) e Verbeeten (2011) destacam, ainda, que deve ser buscado o convencimento prévio dos participantes quanto à relevância da mudança, uma vez que processos dessa natureza somente são exitosos com o comprometimento de todos.

\section{Gestão de custos}

Em cenários de recursos escassos, atualmente comuns tanto a países desenvolvidos como aos do terceiro mundo, metodologias e ferramentas voltadas à otimização do gasto do dinheiro público, como a gestão de custos, se destacam das demais vertentes da NGP. Como destacado por Verbeeten (2011), sistemas de gestão de custos são muito úteis aos gestores políticos, ao apoiarem a tomada de decisão e, ao mesmo tempo, possibilitarem a avaliação de programas e projetos já concluídos, geralmente por meio de benchmarking. Ademais, a gestão de custos se constitui como elemento necessário para a estruturação de outras escolhas estratégicas e gerenciais da NGP (MODELL, 2009).

Os gestores do setor público necessitam, no desempenho de suas funções decisórias, de informações consistentes e amplas para cumprir com eficiência, eficácia e efetividade as políticas públicas (LAPSLEY; WRIGHT, 2004; MACHADO; HolANDA, 2010). Assim sendo, emerge a necessidade da construção de sistemas de gerenciamento de informações de custos que subsidiem as decisões cotidianas, tais como: comprar ou alugar? executar ou terceirizar? manter ou descontinuar? As informações de custos também devem permitir comparações entre os custos de atividades ou serviços produzidos por unidades ou entidades diferentes, objetivando conhecer e estimular a melhoria do desempenho de seus dirigentes (MACHADO; HOLANDA, 2010).

Além disso, as informações de custos associadas aos benefícios das políticas públicas deveriam ser a base para a formulação da proposta orçamentária, sendo o orçamento o fio condutor que permite executar as despesas e prestar os serviços públicos planejados. Ou seja, a informação de custos tem reflexos em todo o ciclo da gestão e, como defendido por Mättö e Sippola (2016), deveria estar presente no cotidiano de todas as entidades governamentais.

Carneiro et al. (2012) levantam uma questão interessante sobre a não tradição de uso da informação de custos no Brasil: "a maioria dos gestores não sabem dizer se as ações oferecidas à sociedade são bem-sucedidas ou não". Esse cenário se 
reflete negativamente em todo o ciclo de gestão, uma vez que dificulta, e algumas vezes inviabiliza, desde a tomada de decisão mais cotidiana até as decisões de alto impacto financeiro ou social (CARNEIRo et al, 2012; FrANCO et al., 2013).

Ressalta-se, todavia, que, assim como as demais mudanças oriundas da NGP, a adoção de ferramentas e estratégias de gestão de custos, bem como a construção de sistemas de informações de custos, além dos benefícios, implicam fatores dificultadores. Além das possíveis explicações listadas por Johansson e Siverbo (2009), várias outras são exploradas em estudos empíricos em organismos estatais ao redor do mundo, objetivando reduzir os percalços a serem enfrentados por gestores e governos que se aventurem em jornadas similares. Neste estudo são brevemente abordados alguns estudos que retratam casos práticos de implantação, e outros que tratam de alguma forma da temática de custos no setor público.

\section{Delineamento metodológico}

O presente estudo se caracteriza como descritivo-exploratório. É exploratório por buscar compreender as experiências de implementação de sistemas ou ferramentas de gerenciamento de custos no setor público ao redor do mundo. E descritivo por apresentar e analisar criticamente os principais achados de alguns estudos internacionais, quanto às dificuldades enfrentadas pelos entes governamentais no processo, bem como os benefícios proporcionados à gestão.

Para o atingimento do objetivo proposto, utilizou-se das técnicas de pesquisa da bibliometria e a análise de conteúdo. A bibliometria, para Macias-Chapula (1998), é um conjunto de leis e princípios empíricos que contribuem para o estabelecimento de fundamentos teóricos. A análise de conteúdo, utilizada na abordagem dos artigos, na perspectiva de Bardin (2004), não se configura como um instrumento, mas como um conjunto de técnicas de análise dos formatos diversos de comunicação.

Os artigos objeto de análise neste estudo foram coletados na base de dados Elsevier Scopus, na qual foram aplicadas as seguintes keywords como filtro de busca: costs, public sector, public management, implant, implement. Foram localizados primariamente 55 trabalhos, sendo que 31 foram excluídos após leitura dos resumos, por não manter relação estreita com o objeto de estudo. Após buscas em bases de dados e na internet, localizaram-se os arquivos de 17 dos 24 trabalhos restantes, sendo esse o conjunto de trabalhos analisados (Quadro 1). 
Quadro 1 - Relação dos trabalhos analisados

\begin{tabular}{|c|c|c|}
\hline Autores & Título & Journal \\
\hline \multicolumn{3}{|c|}{ Artigos que abordam casos de implementação } \\
\hline $\begin{array}{l}\text { Mättö e } \\
\text { Sippola (2016) }\end{array}$ & $\begin{array}{l}\text { Cost management in the public sector: } \\
\text { legitimation behavior and relevant } \\
\text { decision making }\end{array}$ & $\begin{array}{l}\text { Financial } \\
\text { Accountability \& } \\
\text { Management }\end{array}$ \\
\hline $\begin{array}{l}\text { Mello et al. } \\
(2016)\end{array}$ & $\begin{array}{l}\text { Target value design in the public } \\
\text { sector: evidence from a hospital } \\
\text { project in San Francisco, CA }\end{array}$ & $\begin{array}{l}\text { Architectural } \\
\text { Engineering and } \\
\text { Design Management }\end{array}$ \\
\hline Aguilà (2016) & $\begin{array}{l}\text { In search of financial sufficiency in } \\
\text { the Spanish public university: from } \\
\text { financing to the cost control and cost } \\
\text { management }\end{array}$ & Intangible Capital \\
\hline $\begin{array}{l}\text { Gosselin, } \\
\text { Henri e Laurin } \\
\text { (2015) }\end{array}$ & $\begin{array}{l}\text { Costing governmental services in a } \\
\text { reformed environment: unreachable } \\
\text { goal or unfinished business? }\end{array}$ & $\begin{array}{l}\text { Canadian Public } \\
\text { Administration }\end{array}$ \\
\hline $\begin{array}{l}\text { Kosmas e } \\
\text { Dimitropoulos } \\
\text { (2013) }\end{array}$ & $\begin{array}{l}\text { Activity based costing in public sport } \\
\text { organizations: evidence from Greece }\end{array}$ & $\begin{array}{l}\text { Research Journal of } \\
\text { Business Management }\end{array}$ \\
\hline $\begin{array}{l}\text { Zunino, } \\
\text { Borgert e } \\
\text { Schultz (2012) }\end{array}$ & $\begin{array}{l}\text { The integration of benefit-cost ratio } \\
\text { and strategic cost management: the } \\
\text { use on a public institution }\end{array}$ & Revista Espacios \\
\hline $\begin{array}{l}\text { Lutilsky, } \\
\text { Vašiček e } \\
\text { Vašiček (2012) }\end{array}$ & $\begin{array}{l}\text { Cost planning and control in Croatian } \\
\text { public sector }\end{array}$ & $\begin{array}{l}\text { Economic Research } \\
\text { Ekonomska } \\
\text { Istraživanja }\end{array}$ \\
\hline $\begin{array}{l}\text { Verbeeten } \\
\text { (2011) }\end{array}$ & $\begin{array}{l}\text { Public sector cost management } \\
\text { practices in the Netherlands }\end{array}$ & $\begin{array}{l}\text { International Journal } \\
\text { of Public Sector } \\
\text { Management }\end{array}$ \\
\hline \multicolumn{3}{|c|}{ Demais artigos } \\
\hline $\begin{array}{l}\text { Ikeanyibe } \\
\text { (2016) }\end{array}$ & $\begin{array}{l}\text { New public management and } \\
\text { administrative reforms in Nigeria }\end{array}$ & $\begin{array}{l}\text { International } \\
\text { Journal of Public } \\
\text { Administration }\end{array}$ \\
\hline Siltala (2013) & $\begin{array}{l}\text { New public management: the } \\
\text { evidence-based worst practice? }\end{array}$ & $\begin{array}{l}\text { Administration \& } \\
\text { Society }\end{array}$ \\
\hline
\end{tabular}




\begin{tabular}{|c|c|c|}
\hline $\begin{array}{l}\text { Wang e Bryer } \\
\text { (2013) }\end{array}$ & $\begin{array}{l}\text { Assessing the costs of public } \\
\text { participation: a case study of two } \\
\text { online participation mechanisms }\end{array}$ & $\begin{array}{l}\text { The American } \\
\text { Review of Public } \\
\text { Administration }\end{array}$ \\
\hline $\begin{array}{l}\text { Connolly e } \\
\text { Wall (2012) }\end{array}$ & $\begin{array}{l}\text { Implementing IFRSs in the public } \\
\text { sector: caveats from a case in the UK }\end{array}$ & $\begin{array}{l}\text { Public Money \& } \\
\text { Management }\end{array}$ \\
\hline $\begin{array}{l}\text { Meersman } \\
(2010)\end{array}$ & $\begin{array}{l}\text { Applying SMC pricing in PPPs for the } \\
\text { maritime sector }\end{array}$ & $\begin{array}{l}\text { Research in } \\
\text { Transportation } \\
\text { Economics }\end{array}$ \\
\hline $\begin{array}{l}\text { Riemsdijk } \\
(2010)\end{array}$ & $\begin{array}{l}\text { Neoliberal reforms in elder care in } \\
\text { Norway: roles of the state, Norwegian } \\
\text { employers, and Polish nurses }\end{array}$ & Geoforum \\
\hline $\begin{array}{l}\text { Giauque, } \\
\text { Resenterra e } \\
\text { Siggen (2009) }\end{array}$ & $\begin{array}{l}\text { Modernization routes and relations } \\
\text { between political and administrative } \\
\text { spheres in Switzerland }\end{array}$ & $\begin{array}{l}\text { International Review } \\
\text { of Administrative } \\
\text { Sciences }\end{array}$ \\
\hline $\begin{array}{l}\text { Shen e Liu } \\
(2004)\end{array}$ & $\begin{array}{l}\text { Applications of value management in } \\
\text { the construction industry in China }\end{array}$ & $\begin{array}{l}\text { Engineering, } \\
\text { Construction and } \\
\text { Architectural } \\
\text { Management }\end{array}$ \\
\hline Baker (2001) & $\begin{array}{l}\text { Engaging community mental health } \\
\text { stakeholders in pharmacy cost } \\
\text { management }\end{array}$ & Psychiatric Services \\
\hline
\end{tabular}

Fonte: Elaboração própria.

Os trabalhos que abordam casos de implementação de ferramentas de gestão de custos em entes governamentais são apresentados de forma crítica na seção seguinte, dando destaque às principais dificuldades encontradas pelos gestores. Os demais artigos, que abordam a temática sob outra lente e metodologia (levantamentos, ensaios teóricos, entre outros), contribuíram para discussão que se faz também na seção seguinte.

\section{Análise e discussão}

Nesta seção são apresentados os artigos que relatam e analisam casos de implementação de sistemas, metodologias ou ferramentas de gestão de custos em organizações públicas. Também são abordados resultados ou constatações dos demais trabalhos que compõem o conjunto de análise constante no Quadro 1. Com base no arcabouço teórico levantado, também se inicia uma discussão acerca dos fatores relevantes para a temática de custos no setor público. 


\section{Experiências de implantação}

\section{Mättö e Sippola (2016)}

Por meio de uma pesquisa intervencionista direcionada a municípios finlandeses e realizada entre 2007 e 2009, Mättö e Sippola (2016) implementaram um projeto de abordagem colaborativa de gestão dos custos de má qualidade (costs of poor quality - COPQ), de forma a segregar os custos maléficos daqueles que são indispensáveis à consecução dos objetivos organizacionais com a máxima qualidade possível. A intervenção se deu em contratos firmados entre um município finlandês e duas organizações prestadoras de serviços de manutenção de ruas e parques que atuam de forma integrada.

Por meio da intervenção, os autores identificaram gargalos na gestão dos custos organizacionais pela perspectiva bottom up - ou seja, ouvindo os colaboradores desde o nível operacional até os gestores de alto escalão -, de forma a estabelecer, em um segundo momento, estratégias para mitigação dos custos de má qualidade e direcionamento dos gastos governamentais a atividades que realmente contribuam com a qualidade dos serviços postos à disposição da população finlandesa.

$\mathrm{Na}$ perspectiva dos autores, além da otimização dos gastos públicos, a implementação da ferramenta de gestão elevou a qualidade das informações postas à disposição dos decisores, uma vez que mapeia de forma mais ampla e fidedigna a destinação dos recursos, bem como os resultados dos programas governamentais. Esse fator se reflete, na percepção dos gestores participantes do processo, na legitimação de suas atividades perante a sociedade.

Ao final do processo, os autores destacaram como principal entrave a superestimação das funcionalidades da ferramenta e a subestimação dos problemas que poderiam surgir. Ou seja, os colaboradores e gestores esperavam que o campo de impacto da nova metodologia de gestão de custos fosse maior, e que esta não causaria novas demandas de trabalho. Outros dificultadores, segundo os autores, foram: não acompanhamento e avaliação sequencial do processo, resistência por parte dos colaboradores para alterações de seus protocolos internos e insuficiência de recursos para desenvolvimento de projetos conexos na sequência (JoHANSSON; SIVERBO, 2009).

\section{Mello et al. (2016)}

O estudo de Mello et al. (2016) foi realizado no Hospital da Universidade da Califórnia, localizado na cidade de São Francisco (EUA). Os autores acompanharam a adoção do método target value design -TVD (projeto de valor alvo) no planejamento 
integrado e execução de um complexo de edifícios do hospital, entre os anos de 2010 e 2015. O TVD, por meio da metodologia do custeio-meta, objetiva entregar o produto ao cliente pelo valor que ele pretende pagar (BALLARD, 2011).

No caso pesquisado, a gestão do hospital fixou o preço de construção do complexo de acordo com as normas legais, que se baseiam em índices e pesquisas de mercado. Na sequência, reduziu o preço fixado anteriormente em 15\%, definindo este como o valor máximo a ser despendido pelas obras (o valor alvo). Assim sendo, a empreiteira parceira e a equipe do órgão público tiveram a missão de fazer adequações nos projetos e no planejamento de forma a manter o objeto principal (complexo hospitalar), todavia com um orçamento 100 milhões de dólares inferior ao praticado no mercado.

Os autores identificaram como benefícios oriundos da utilização da ferramenta de gestão de custos a redução dos custos e a otimização dos projetos, uma vez que se buscaram alternativas de melhoria a todo o tempo, de forma a se manterem dentro do orçamento. Outro ponto positivo percebido foi a motivação dos participantes do projeto, em virtude do estabelecimento de metas e desafios contemplados com premiações. Ademais, os gestores perceberam a ampliação da integração entre a equipe, que se tornou mais capacitada e apta à adoção de outras ferramentas gerenciais.

Como principal fator dificultador do processo de implementação do TVD no setor público, os autores elencaram a limitação normativa para firmar contratos de múltiplas partes. Esse tipo de instrumento contratual amplia os benefícios do TVD, ao dividir riscos e perdas entre a entidade governamental e as demais signatárias, induzindo as partes a galgar oportunidades mais vantajosas e arriscadas. Essa barreira normativa, na visão de Gosselin, Henri e Laurin (2015), se constitui devido à coexistência de duas lógicas institucionais em processos de transição. Uma legalistaburocrática e outra gerencial, sendo que, via de regra, a primeira inviabiliza ações da segunda.

\section{Kosmas e Dimitropoulos (2013)}

Em um estudo de caso, Kosmas e Dimitropoulos (2013) acompanharam a aplicação do custeio baseado em atividades (activity based costing) em uma entidade pública desportiva localizada na cidade de Papagos-Halargos, Grécia. Tal organização, que disponibiliza uma série de atividades esportivas à população local, não dispunha de nenhum método definido de levantamento e controle de custos e, para mensurar seu desempenho, dividia os gastos totais pelo número de atividades realizadas. $A$ adoção da metodologia $A B C$, segundo os autores, emergiu da crise 
que a Grécia passa nos últimos anos, obrigando os gestores a buscarem meios de reduzirem os gastos governamentais.

Os resultados da aplicação do activity based costing trouxeram à tona dados preocupantes aos gestores: atividades atléticas componentes do objeto da entidade, consideradas irrelevantes no processo de controle de gastos (pelo método tradicional), apresentaram alta participação na destinação dos recursos recebidos pela organização. Ou seja, grande parte do orçamento vinha sendo consumido por um número reduzido de atividades.

De posse das informações de custos por atividades executadas, os gestores gregos, em sua própria percepção, estiveram melhor preparados para a construção do planejamento para os exercícios seguintes e puderam avaliar a (des)continuidade de alguns serviços. Kosmas e Dimitropoulos (2013) concluíram que a inserção de método $A B C$ na gestão pública local foi simplificada, todavia não trouxe avanços significativos em relação a outros métodos de custeio. Segundo os autores, a falsa sensação percebida pelos gestores é reflexo de não praticarem anteriormente nenhuma forma de gestão de custos.

Nesse cenário, os autores apontam que a preparação e capacitação insuficiente dos gestores é um dos principais fatores causadores dos inúmeros problemas que permeiam a administração pública, e que dificultam o seu desenvolvimento. Essa posição vai ao encontro do defendido por Malmi (1997).

\section{Verbeeten (2011)}

Em questionário encaminhado a gestores e controllers de 57 entidades governamentais da Holanda, Verbeeten (2011) objetivou identificar e mapear as principais metodologias de gerenciamento de custos adotadas pelas instituições, a motivação de sua implementação e a forma, nível e intensidade do uso da informação gerada pelos sistemas gerenciais.

O autor constatou a predominância do reconhecimento total dos custos aos serviços prestados. Ou seja, sem a distinção entre custos diretos, indiretos e despesas, e sem a adoção de critérios de rateio, como departamentalização ou por atividades. Mesmo sendo citados alguns métodos de custeio mais robustos, como o baseado em atividades, via de regra os entes governamentais holandeses se utilizam de métodos simplificados.

Quanto às informações de custos, constatou-se que sua utilização está vinculada à percepção de sua relevância e eficácia para o processo decisório. Os gestores que afirmaram usá-las com maior frequência foram os vinculados a áreas financeiras, enquanto que os gestores políticos apresentam um uso pequeno da informação, 
mesmo tomando decisões frequentemente. Esse cenário vai ao encontro da posição defendida por Ter Bogt (2008), de que os gestores políticos baseiam grande parte de suas decisões em posicionamentos de seus assessores técnicos.

O levantamento de Verbeeten (2011) detectou ainda que a motivação para implementação de quaisquer ferramentas de gestão de custos emerge do ambiente externo (sociedade) e estas são construídas visando à legitimação de curto prazo da entidade por meio do controle da destinação dos recursos públicos. Não há, todavia, a indicação de uso das informações e ferramentas de custos em ações de longo prazo vinculadas ao planejamento estratégico.

\section{Zunino, Borgert e Schultz (2012)}

O presente estudo foi realizado em solo nacional. Mais especificamente, no restaurante universitário da UFSC. Zunino, Borgert e Schultz (2012) integraram a análise do índice de benefício-custo na gestão estratégica de custos do restaurante, buscando identificar o status quo do desempenho sob a ótica social. $\mathrm{O}$ índice benefício-custo encontrado foi de 0,89, o que representa um indicativo de inviabilidade econômica. Ou seja, o custo dos serviços prestados foi superior aos benefícios à comunidade acadêmica da UFSC.

Os autores realizaram diversas simulações em cenários distintos, como a terceirização dos serviços, a limitação dos beneficiados e ampliação do volume produzido. Na maioria das simulações encontraram possibilidades mais viáveis economicamente para a instituição.

Nesse contexto, em que foram propostas mudanças significativas na estrutura, funcionamento e abrangência do restaurante universitário, a pressão política pela manutenção dos benefícios se sobrepõe aos avanços na gestão, assim como argumentado por Gosselin, Henri e Laurin (2015).

\section{Aguilà (2016)}

Aguilà (2016), diante da política orçamentária restritiva nos países da União Europeia, analisou canais alternativos para financiamento das universidades públicas espanholas, bem como possíveis técnicas de controle e gestão de custos que, se implementadas, contribuiriam para a manutenção das universidades no cenário de crise.

Com relação às técnicas de controle e gestão de custos, o autor fez um levantamento junto aos gestores das universidades públicas da Espanha, objetivando captar suas percepções sobre algumas metodologias e sobre os benefícios à gestão propostos por cada técnica. Ficou evidenciada a consciência dos gestores quanto à 
eficácia das técnicas e quanto à necessidade de se adotá-las no atual contexto. Essa percepção das contribuições da técnica, conforme defendido por Ter Bogt (2008) e Verbeeten (2011), é um dos quesitos mais relevantes para o sucesso da empreitada.

Todavia, os gestores defendem que o próprio cenário de crise que cria a necessidade de melhor gerenciamento do dinheiro público o torna mais difícil. Isso porque se fazem necessários cortes de pessoal, redução de jornada de trabalho, suspensão de capacitação profissional, entre outros fatores que impactam diretamente a capacidade de gerenciamento organizacional. Em suma, os gestores universitários entendem que o contexto sócio-econômico reflete diretamente na gestão e nos resultados das entidades.

\section{Gosselin, Henri e Laurin (2015)}

Diante de pressões externas sociais em virtude do aumento de tarifas de serviços cartoriais, a gestão de um órgão descentralizado canadense decidiu implementar um sistema de custeio, com o qual pudesse mensurar os custos dos diferentes serviços prestados e justificar possíveis ajustamentos das tarifas. Gosselin, Henri e Laurin (2015), por meio de pesquisa-ação em parceria com os gestores, desenvolveram um modelo de custeio $A B C$ de quatro etapas.

$\mathrm{Na}$ análise das informações geradas pelo sistema $A B C$, ficou evidenciado que os dois principais serviços prestados pela entidade estavam sendo operados em déficit. Ou seja, os custos para sua execução eram superiores ao valor tabelado cobrado pelo serviço. Com base nessa análise primária, os gestores concluíram que essas duas atividades deficitárias foram a causa, até então desconhecida, do resultado negativo do exercício anterior.

Os autores perceberam que, mesmo ficando evidenciados os benefícios da informação de custos para a gestão, os gestores não demonstraram sinais de aumento da consciência de custos. Ou seja, na percepção dos gestores, o sistema de custos adotado serviu como um meio para legitimar o aumento das tarifas perante as pressões externas, e não como uma técnica que torna a gestão mais eficiente (IKEANYIBE, 2016). O cenário apresentado se alinha a uma das possíveis explicações para o não sucesso de reformas no setor público elencadas por Johansson e Siverbo (2009).

\section{Lutilsky, Vašiček e Vašiček (2012)}

Por meio de questionários enviados a gestores públicos croatas, de diversas entidades e em períodos distintos (2006 e 2011), Lutilsky, Vašiček e Vašiček (2012) objetivaram identificar a evolução das práticas gerenciais relacionadas à gestão de custos adotadas pelos entes. A motivação do estudo se deu pelo processo de 
reforma governamental que a Croácia iniciou em 2002 e que se encontrava em curso no período estudado.

Os autores identificaram ligeiro aumento da utilização de ferramentas gerenciais relacionadas à gestão de custos nos cinco anos, que possivelmente foi motivado pelas outras ações da reforma governamental, que exigiam informações de custos para sua implementação, conforme defendido por Modell (2009).

Destacam os autores que a principal ameaça ao atingimento de sucesso nas reformas consiste na descontinuidade dos projetos, em virtude de mudanças políticas de governo. Isso porque as ferramentas contábeis geram reflexos a longo prazo, uma vez que se desenvolvem juntamente com o conhecimento adquirido pelos seus praticantes. O posicionamento dos autores corrobora as percepções de Ikeanyibe (2016), Connolly e Wall (2012) e Verbeeten (2011).

Os autores ainda listam outros riscos para o êxito das reformas: falta de integridade da gestão (SILTALA, 2013), decisões inadequadas da alta gestão buscando legitimação (VERBEETEN, 2011), falta de políticas definidas e claras (IKEANYIBE, 2016; Kosmas; Dimitropoulos, 2013; Verbeeten, 2011) e ausência de valores éticos (IKEANYIBE, 2016; SILTALA, 2013).

\section{Discussão}

Na leitura dos achados internacionais, percebem-se similaridades com relação às experiências das entidades governamentais em processos de implementação de ferramentas de gestão de custos, mesmo sendo analisados casos de países de várias regiões do mundo (América do Norte, América do Sul, leste e centro europeus, ibéricos, nórdicos). Tais similaridades refletem as características próprias do setor público, que resistem na administração independentemente das estratégias de gestão de cada país (LAPSLEY; WRIGHT, 2004; MODELL, 2009).

Nesse sentido, as dificuldades e riscos de não atingimento de sucesso nos projetos também se alinham e podem ser sintetizados de forma agrupada como segue.

\section{Foco na legitimação}

A gestão de custos foi constituída visando à otimização da eficiência das organizações privadas e públicas, e este deveria ser o motivador de sua adoção em qualquer modelo de gestão (LAPSLEY; WRIGHT, 2004). Todavia, como verificado nos estudos de Kosmas e Dimitropoulos (2013), Verbeeten (2011), Gosselin, Henri e Laurin (2015) e Mättö e Sippola (2016), os administradores públicos têm desviado o foco das ferramentas, e as utilizado como meio para legitimar politicamente sua gestão perante os usuários dos serviços, empresas, outros órgãos públicos e a sociedade como um todo. Esses cenários confirmam o já constatado por Modell (2009). 
Vale ressaltar que a busca por legitimação em si não se configura como um problema organizacional. Todavia se torna um quando suprime ou se sobrepõe em desfavor de outras necessidades institucionais.

\section{Não integração}

Sistemas de gestão de custos são componentes de outros sistemas estruturantes da organização e, sozinhos, não atingem um nível elevado de eficiência. Ademais, outros artefatos gerenciais também necessitam do sistema de custos para atingimento de seus objetivos (MODELL, 2009). Dessa forma, a não vinculação de ferramentas de custos com o planejamento institucional, consignando suas relações e o processo de manutenção e avaliação, se configura como um risco de fracasso ao processo de implementação. Tais cenários foram detectados nos estudos de Mättö e Sippola (2016), Kosmas e Dimitropoulos (2013), Verbeeten (2011), Lutilsky, Vašiček e Vašiček (2012) e Zunino, Borgert e Schultz (2012).

\section{Insuficiência de recursos}

Cenários permeados por crises econômicas servem, ao mesmo tempo, para motivar a adoção de ferramentas de custos (buscando a redução de despesas) como para inviabilizar a implementação. Isso ocorre devido à necessidade de investimento em pessoas e infraestrutura para consecução de projetos dessa natureza. Mesmo não relatando ocorrências de projetos interrompidos por problemas financeiros, alguns autores relataram a percepção de indícios de descontinuidade por esse motivo (Gosselin; HenRI; LAURIN, 2015; MÄTtÖ; SiPPOlA, 2016; Kosmas; Dimitropoulos, 2013; ZUNINO; BORGERT; SCHULTZ, 2012).

\section{Normas regulamentares}

Em virtude de princípios do direito público, sobretudo da legalidade, a administração governamental atua em um contexto amplamente normativo. Por esse motivo, não dispõe de discricionariedade na maioria de seus atos. Nesse sentido, há o comprometimento de parte das funcionalidades das técnicas importadas da iniciativa privada, devido à impossibilidade de adoção de algumas práticas. Dificuldades dessa natureza foram citadas em todos os trabalhos, porém o estudo de Mello et al. (2016) apresentou maior impacto.

\section{Considerações finais}

O presente trabalho teve como objetivo investigar experiências de implementação de sistemas de gestão de custos no setor público em âmbito internacional, de forma 
a identificar as principais dificuldades enfrentadas pelos entes governamentais no processo, bem como os benefícios proporcionados à gestão.

Por meio da análise de 17 trabalhos internacionais, que apresentam casos de implementação de ferramentas de gestão de custos em entes governamentais ou abordam de alguma forma a temática, foram extraídos os principais achados dos autores quanto ao andamento do processo, as dificuldades encontradas e os benefícios percebidos à gestão pública.

Os resultados indicam similaridade entre os fatores influenciadores do processo de implementação nos diversos países, mesmo havendo heterogeneidade no conjunto analisado. As dificuldades mais recorrentes foram: a) foco na legitimação e não na eficiência, b) não integração da ferramenta com os demais sistemas estruturantes e com o planejamento estratégico, c) escassez de recursos para iniciar e dar continuidade aos projetos e d) excesso normativo que inviabiliza algumas ações. Nesse cenário, os achados de Johansson e Siverbo (2009) se confirmam.

O foco na legitimação, conforme corroborado pelos estudos anteriores de Modell (2009), Verbeeten (2011) e Mättö e Sippola (2016), é um fator altamente impactante nas reformas da NGP e, sobretudo, na adoção de sistemas de gestão custos. 0 isomorfismo coercitivo induz frequentemente gestores a um comportamento focado na busca de legitimação, seja para estar em conformidade com modelos ideais, seja para moldar sua imagem política perante a sociedade. Segundo Dimaggio e Powell (1983), organizações tendem a ceder a pressões externas, explícitas ou implícitas, de forma coercitiva, em busca de aceitação ou legitimidade.

Pode-se concluir que, assim como no Brasil, a administração pública de alguns países ainda carece de desenvolvimento em sua estrutura de gestão estratégica de custos, uma vez que fica evidenciado, pela literatura e pelas experiências de alguns países, (LAPSLEY; Wright, 2004; MOdelL, 2009; TER BOGT, 2008 entre outros) o estágio ainda embrionário em que a mesma se encontra. Assim como argumentado por Gosselin, Henri e Laurin (2015), o movimento de reformas da NGP depende, para seu sucesso, de uma mudança nas lógicas institucionais, deixando a perspectiva burocrática para trás e absorvendo o caráter gerencial. Nesse cenário, a consciência de custos, tão relevante para sua efetividade, emerge naturalmente.

Diante do exposto até aqui, quando se pôde conhecer um pouco das experiências de alguns países, entende-se que a pergunta de pesquisa foi respondida e que os resultados encontrados podem ser úteis a gestores e servidores públicos que pretendam iniciar um projeto de implantação. As informações aqui apresentadas podem tornar menos sinuoso o caminho em busca da eficiência na gestão dos recursos públicos. 
Como limitação deste artigo se destaca a não localização do arquivo digital de alguns artigos selecionados na busca. Para pesquisas subsequentes, sugerese a realização de estudos de casos múltiplos, de forma a possibilitar maior aprofundamento na busca das raízes das dificuldades inerentes ao setor público.

\section{Referências bibliográficas}

AGUILÀ, S. In search of financial sufficiency in the Spanish public university: from financing to the cost control and cost management. Intangible Capital, v. 12, n. 1, p. 17-50, 2016.

Alonso, M. Custos no serviço público. Revista do Serviço Público, v. 50, n. 1, p. 3763, 1999.

BALLARD, Glenn. Target value design: current benchmark (1.0). Lean Construction Journal, v. 2011, p. 79-84, 2011.

BARDIN, L. Análise de conteúdo. Lisboa: Edições 70, 2004.

BittI, E. J. S.; Aquino, A. C. B.; CARDoso, R. L. Adoção de sistemas de custos no setor público: reflexões sobre a literatura nacional veiculada em periódicos acadêmicos. Revista Universo Contábil, v. 7, n. 3, p. 06-24, 2011.

CONNOLLY, C.; WALL, A. Implementing IFRSs in the public sector: caveats from a case in the UK. Public Money \& Management, v. 32, n. 1, p. 53-60, 2012.

CARdoso, R. L.; Aquino, A. C. B.; BITTI, E. J. S. Reflexões para um framework da informação de custos do setor público brasileiro. Revista Administração Pública, v. 45, n. 5, p. 1565-1586, 2011.

CARNEIRO, A. F. SILVAet al. Custos na administração pública: revisão focada na publicação de artigos científicos a partir da promulgação da Lei de Responsabilidade Fiscal. Revista Contemporânea de Contabilidade, v. 9, n. 18, p. 3-22, 2012.

BRASIL. Lei Complementar n. 101/2.000. Estabelece normas de finanças públicas voltadas para a responsabilidade na gestão fiscal e dá outras providências. Disponível em: <http://www.planalto.gov.br/ccivil_03/leis/lcp/lcp101.htm>.

Dimaggio, P. J.; POWELL, W. W. The iron cage revisited-institutional isomorphism and collective rationality in organizational fields. American Sociological Association, v. 48, n. 2, p. 147-160, 1983.

FRANCO, L. M. G. et al. Sistemas de custos: importância, viabilidade e utilidade na concepção dos agentes públicos do Estado do Paraná (Brasil). Revista de Educação e Pesquisa em Contabilidade, v. 7, n. 2, p. 167-183, 2013.

GoSSELIN, M.; HENRI, J.; LAURIN, C. Costing governmental services in a reformed environment: unreachable goal or unfinished business? Canadian Public Administration, v. 58, n. 3, p. 384-405, 2015.

IKEANYIBE, O. M. New public management and administrative reforms in Nigeria. International Journal of Public Administration, v. 39, n. 7, p. 563-576, 2016. 
JOHANSSON, T.; SIVERBO, S. Explaining the utilization of relative performance evaluation in local government: a multi-theoretical study using data from Sweden. Financial Accountability \& Management, v. 25, n. 2, p. 197-224, 2009.

Kosmas, I. D.; Dimitropoulos, P. E. Activity based costing in public sport organizations: evidence from Greece. Research Journal of Business Management, v. 8, n. 2, p. 130-138, 2013.

LAPSLEY I.; WRIGHT, E. The diffusion of management accounting innovations in the public sector: a research agenda. Management Accounting Research, v. 15, n. 3, p. 355-374, 2004.

Lovato, E. L. et al. Cost information systems in the public sector in Brazil: an analysis of user perception under the focus of government management. Journal Globalization, Competitiveness and Governability, v. 10, n. 3, p. 16-41, 2016.

MACHADO, N. ; HolandA, Victor Branco. Diretrizes e modelo conceitual de custos para o setor público a partir da experiência no governo federal do Brasil. Revista de Administração Pública, v. 44, n. 4, p. 791-820, 2010.

MACIAS-CHAPULA, C. A. O papel da informetria e da cienciometria e sua perspectiva nacional e internacional. Ciência da Informação, v. 27, n. 2, p. 134-140, 1998.

MAGRo, D.; PINTO, M. D. S. Os efeitos da nova gestão pública na produção de conhecimento científico. Navus-Revista de Gestão e Tecnologia, v. 2, n. 2, p. 78-89, 2012.

MALMI, T. Towards explaining activity-based costing failure: accounting and control in a decentralized organization. Management Accounting Research, v. 8, n. 4, p. 459-480, 1997.

MÄTTÖ, T.; SIPPOLA, K. Cost management in the public sector: legitimation behaviour and relevant decision making. Financial Accountability \& Management, v. 32, n. 2, p. 179-201, 2016.

MELLO, R. S. S. et al. Target value design in the public sector: evidence from a hospital project in San Francisco, CA. Architectural Engineering and Design Management, v. 12, n. 2, p. 125-137, 2016.

MODELL, S. Institutional research on performance measurement and management in the public sector accounting literature: a review and assessment. Financial Accountability \& Management, v. 25, n. 3, p. 277-303, 2009.

MotTA, P. R. M. O estado da arte da gestão pública. Revista de Administração de Empresas, v. 53, n. 1, p. 82-90, 2013.

OSBORNE, D.; GAEBLE, T. Reinventing government: how the entrepreneurial spirit is transforming the public sector. New York: Penguin Group, 1992.

LUTILSKY, I. D.; VAŠIČEK, V.; VAŠIČEK, D. Cost planning and controlin Croatian public sector. Economic Research-Ekonomska Istraživanja, v. 25, n. 2, p. 413-434, 2012.

SILTALA, J. New public management the evidence-based worst practice? Administration \& Society, v. 45, n. 4, p. 468-493, 2013.

TER BOGT, H. J. Management accounting change and new public management in local government: a reassessment of ambitions and results - an institutionalist 
approach to accounting change in the Dutch public sector. Financial Accountability \& Management, v. 24, n. 3, p. 209-241, 2008.

Verbeeten, F. H. M. Public sector cost management practices in the Netherlands. International Journal of Public Sector Management, v. 24, n. 6, p. 492-506, 2011. ZUNINO, A.; BORgERT, A.; SCHULTZ, C. A. The integration of benefit-cost ratio and strategic cost management: the use on a public institution. Espacios, v. 33, p. 1-2, 2012.

\section{Diego Messias}

Mestre em Contabilidade pela Universidade Estadual do Oeste do Paraná (Unioeste). Atualmente é contador da Universidade Federal da Integração Latino-Americana (Unila). Contato: siquiasom@gmail.com

\section{Júlio César Ferreira}

Doutorando em Ciências Contábeis pela Universidade do Vale do Rio dos Sinos (Unisinos). Mestre em Contabilidade pela Universidade Estadual do Oeste do Paraná (Unioeste). Atualmente é Professor Assistente da Universidade Estadual do Oeste do Paraná (Unioeste). Contato: juliocesar300@hotmail.com

\section{Dione Olesczuk Soutes}

Doutora em Controladoria e Contabilidade pela Universidade de São Paulo (USP). Atualmente é Professora Adjunta Universidade Estadual do Oeste do Paraná (Unioeste). Contato: dioneosoutes@gmail.com 